\title{
Energy saving through LED in signaling functions for automotive exterior lighting
}

Alexis Bony, Khaled Hamami, Frank Tebbe, Jens Mertens

Alexis Bony, Khaled Hamami, Frank Tebbe, Jens Mertens, "Energy saving through LED in signaling functions for automotive exterior lighting," Proc. SPIE 8065, SPIE Eco-Photonics 2011: Sustainable Design, Manufacturing, and Engineering Workforce Education for a Green Future, 806502 (20 April 2011); doi: $10.1117 / 12.884472$

SPIE. Event: SPIE Eco-Photonics, 2011, Strasbourg, France 


\title{
Energy saving through LED in signaling functions for automotive exterior lighting
}

\author{
Alexis Bony, Khaled Hamami, Frank Tebbe, Jens Mertens \\ Daimler AG; Mercedes-Benz Technologie Center \\ 71059 Sindelfingen, Germany
}

\begin{abstract}
Safety considerations have always driven the way for improving exterior automotive lighting legal requirements. With the recent adoption of day-time running lamps for passenger cars, the steadily increasing need for reduction of vehicle power consumption has led to the introduction of LED-based day-time running lamps.

Solutions with incandescent bulbs have also been implemented, as they present price advantages while offering limited design perspectives. In the meantime, technology developments has turned LED sources into ideal candidates for daytime running lamps by increasing their lumen per watt efficiency ratio towards values around $100 \mathrm{~lm} / \mathrm{W}$ or higher.

In this work, taking as an example the new Mercedes-Benz roadster SLK (R172), we present the first single LED daytime-running lamp, with a total power consumption below $5 \mathrm{~W}$ per vehicle. After reviewing legal requirements, the optical and electronic concepts are discussed.

Details on the tail lamp LED functions are also discussed, and particularly the advantages from the realization of fog lamp with LEDs.
\end{abstract}

Keywords: automotive lighting, exterior lighting, LED, day-time running lamp, tail lamp, energy saving

\section{INTRODUCTION}

Automotive exterior lighting functions may be divided into two groups: illuminating and signaling functions. To the former belong functions that provide the driver with adequate vision conditions during night drive, like low beam and high beam, together with their dynamic horizontal or vertical moves or with their adaption to specific driving situations (highway light, bad weather light,...). To the latter belong functions that ensure the driver is visible for other traffic participants, like tail lamp, stop lamp, or turn indicator.

Figure 1 shows an overview of a possible arrangement of these exterior lighting functions.

SPIE Eco-Photonics 2011: Sustainable Design, Manufacturing, and Engineering Workforce Education for a Green Future, edited by Pierre Ambs, Dan Curticapean, Claus Emmelmann, Wolfgang Knapp, Zbigniew T. Kuznicki, Patrick P. Meyrueis, Proc. of SPIE Vol. 8065, 806502 - (c) 2011 SPIE · CCC code: 0277-786X/11/\$18 - doi: 10.1117/12.884472 


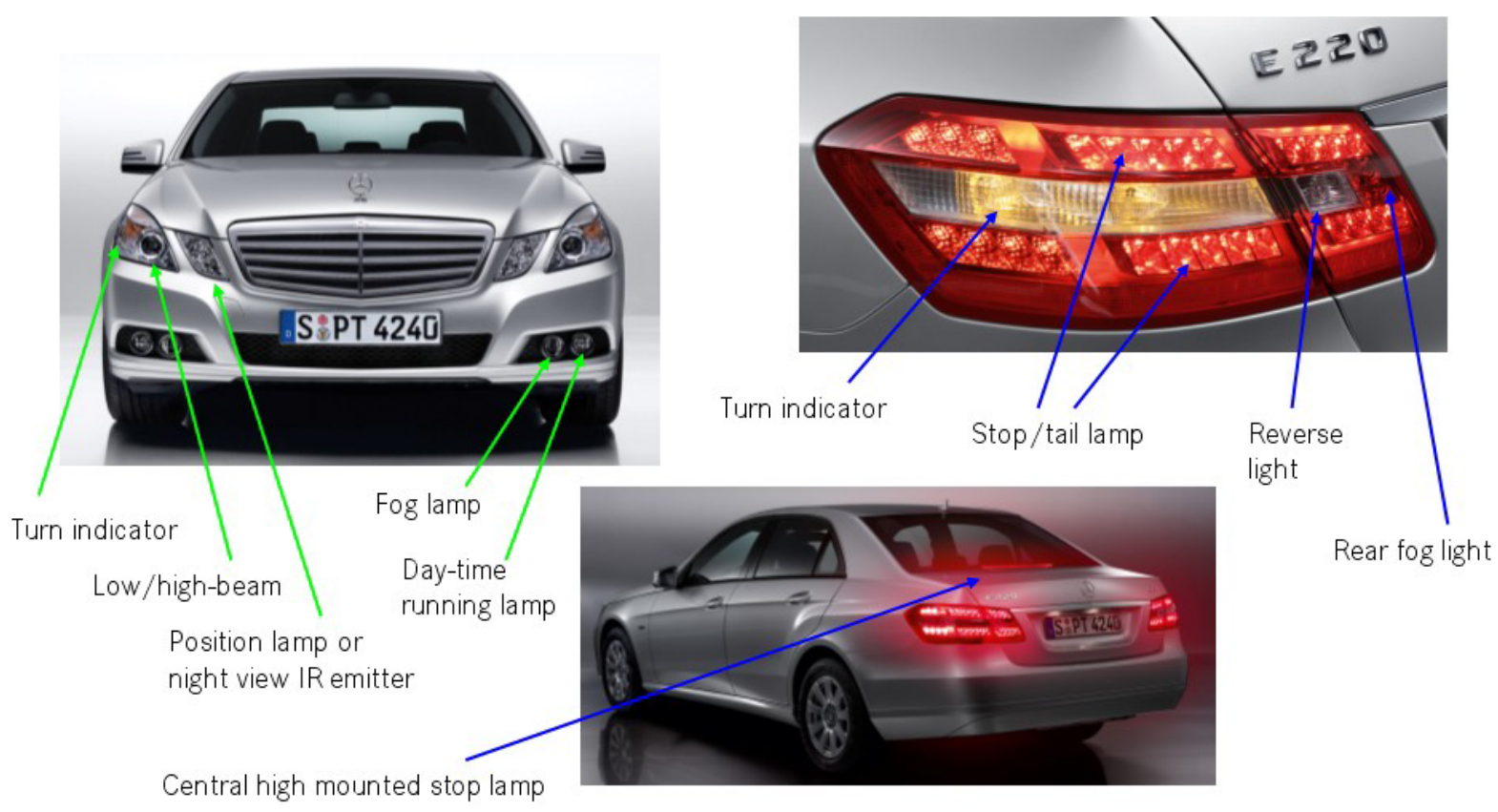

Figure 1: Overview of exterior lighting function arrangement for Mercedes-Benz E-Class

Several calculations were performed for converting power consumption of a given function to fuel quantities or $\mathrm{CO}_{2}-$ emission amount. In order to do so, assumptions have to be formulated on the statistical use of each function, on the car engine and alternator efficiency, as well as number of driven kilometers for a given period. Discussion on this subject would exceed the scope of this paper. The interested reader can refer to cited references ${ }^{1,2}$ for detailed calculations.

We shall however retain from these studies that power consumption reduction can be achieved through using light emitting diodes (LED) for automotive exterior lighting applications, and particularly that signaling functions with higher statistical working time could bring significant contributions in terms of energy saving. Day-time running lamp (DRL) is a new signaling function introduced by the Economic Commission for Europe (ECE) as mandatory for new car models starting from 2011. This lamp should enhance visibility conditions and thus reduce the yearly number of fatalities on traffic roads. This function is always turned on during day-time, what turns it into an ideal candidate for power reduction due to its comparatively long statistical time of use.

There are presently two technological types of light sources available to realize exterior lighting signaling functions and particularly day-time running lamps. Incandescent bulbs have been in use in the automotive industry for several decades ${ }^{3}$ and consist in a metal filament inside a glass bulb that is heated up with a current till light emission. A large part of the input energy is radiated in the infra-red domain, what leads to moderate luminous efficiency below $201 \mathrm{~m} / \mathrm{W}$ for white color. In addition, when a red function like stop lamp is to be realized, color filtering of the incandescent source leads to visible light absorption of the order of approximately $75 \%$.

LEDs are semiconductor based light sources ${ }^{4}$ that emit light when a current flows through the PN-junction. Depending on the color aimed at, light may be used directly from a semiconductor die (yellow, red), or converted via fluorescence through a phosphor layer (mainly for white, but also yellow). Efficiencies starting around $20-30 \mathrm{~lm} / \mathrm{W}$ for yellow and red can reach nowadays up to $100 \mathrm{~lm} / \mathrm{W}$ for white.

Day-time running lamps that are already on the market show three different trends. First trend consists in an incandescent bulb based lamp. With focus on affordable technical solutions and simple design, incandescent bulb candidates may be for instance P21W, H15, P13 or PSX26W. They present as mentioned $1 \mathrm{~m} / \mathrm{W}$ efficiencies below $201 \mathrm{~m} / \mathrm{W}$, and their time of life may not cover that of a passenger car. Depending on the type of lamp selected, total power consumption for a daytime running lamp of first trend may reach between 30 and 50W per vehicle. 
Second trend consists in an LED-based lamp. Parts available today present a reduced power consumption in comparison to first trend solutions, with values between 10 and $20 \mathrm{~W}$ per vehicle, but also a more sophisticated design, using daytime running lamp as a way of brand differentiation. System costs for second trend solutions are often higher than for first trend ones due to system complexity.

In what follows, we introduce what we believe to be a third trend for day-time running lamp. The proposed system makes use of a single LED (but generally speaking of a reduced number of LEDs) to further reduce total power consumption per vehicle below $5 \mathrm{~W}$ while keeping system complexity and thus costs as low as possible.

\section{OPTICAL DESIGN}

Legal requirements for day-time running lamp ${ }^{5}$ (but generally speaking also for signaling functions) are expressed in luminous intensity, that-is-to-say in a quantity of light normalized with averaged human eye sensitivity and divided by a given solid angle. The commonly used unit is candela. Typical requirements consider horizontal and vertical angles departing from the main car direction and define minimum values of intensity for a grid of angles.

Figure 2 shows the vertical and horizontal limit angles for day-time running lamps.
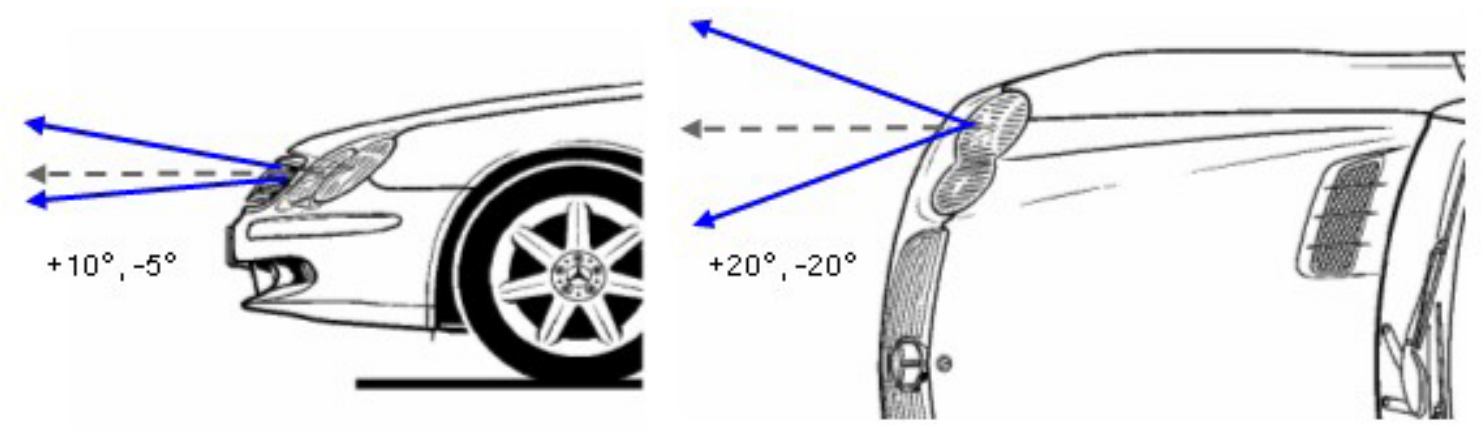

Figure 2: Horizontal and vertical limit angles for day-time running lamp

Figure 3 illustrates a typical minimum value grid for luminous intensity, covering both ECE and SAE (Society of automotive engineers) regulations and including a 10\%-margin.

\begin{tabular}{|c|c|c|c|c|c|c|c|c|c|c|c|}
\hline & $25^{\circ}$ & $20^{\circ}$ & $15^{\circ}$ & $10^{\circ}$ & $5^{\circ}$ & $0^{\circ}$ & $5^{\circ}$ & $10^{\circ}$ & $15^{\circ}$ & $20^{\circ}$ & $25^{\circ}$ \\
\hline $15^{\circ}$ & 0 & 0 & 0 & 0 & 0 & 0 & 0 & 0 & 0 & 0 & 0 \\
\hline $10^{\circ}$ & 0 & 0 & 0 & 0 & 88 & 0 & 88 & 0 & 0 & 0 & 0 \\
\hline $5^{\circ}$ & 0 & 44 & 0 & 110 & 0 & 385 & 0 & 110 & 0 & 44 & 0 \\
\hline $0^{\circ}$ & 0 & 110 & 0 & 308 & 495 & 550 & 495 & 308 & 0 & 110 & 0 \\
\hline $5^{\circ}$ & 0 & 44 & 0 & 110 & 0 & 385 & 0 & 110 & 0 & 44 & 0 \\
\hline $10^{\circ}$ & 0 & 0 & 0 & 0 & 0 & 0 & 0 & 0 & 0 & 0 & 0 \\
\hline
\end{tabular}

Figure 3: DRL luminous intensity requirements

The purpose of this work was to develop a part with the lowest achievable power consumption. Consequently, the optical concept was chosen so as to maximize optical efficiency (here defined as light gathered by the optical system) in order to be able to use a single LED.

Figure 4 represents a section cut of the optical system and shows the working principles of the double reflector. 


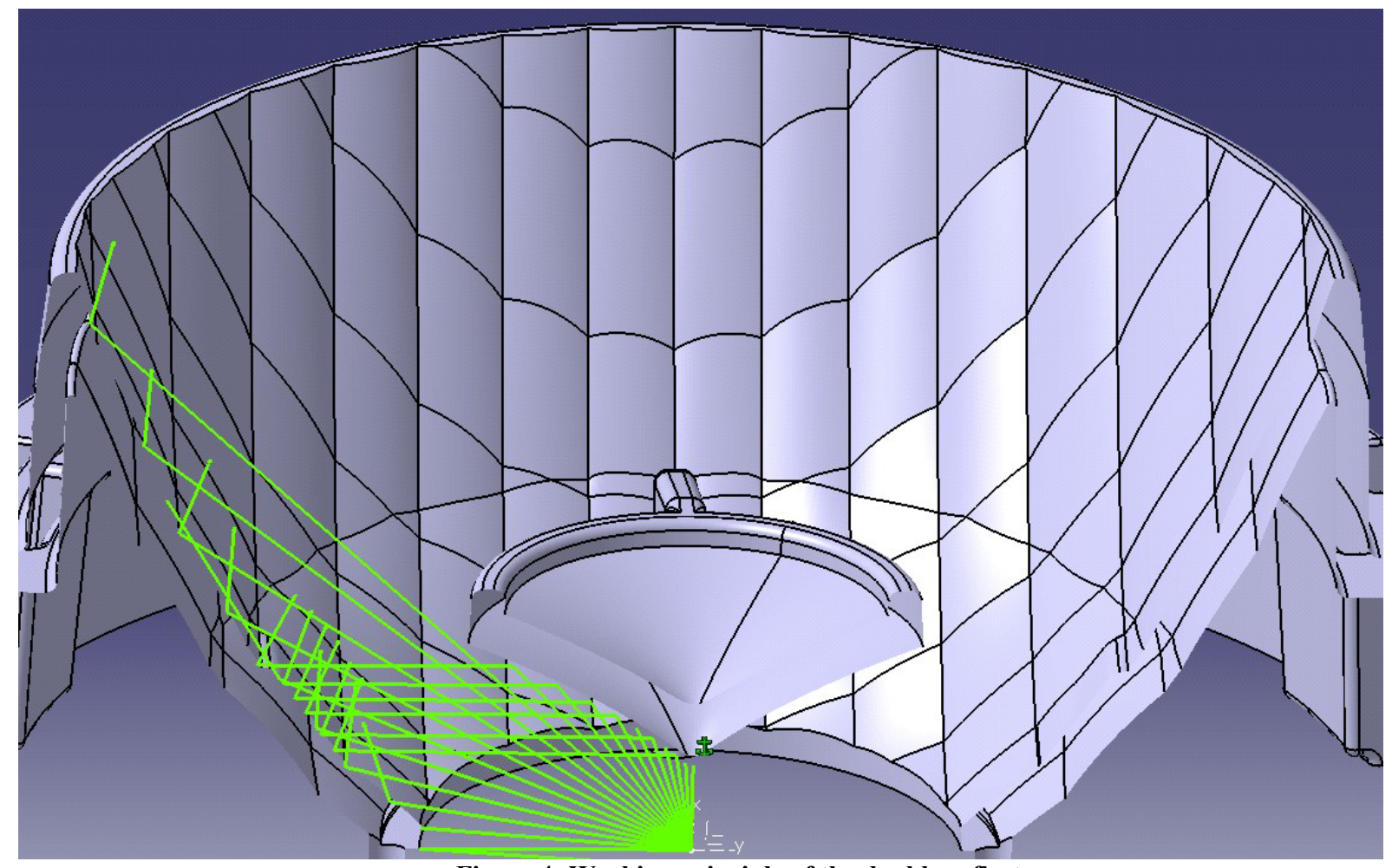

Figure 4: Working principle of the double reflector

Light emitted from the LED, here replaced by a point source, can be divided into two parts. The first part is collected directly by the side reflector and then scattered by a curved surface, often called "pillow", or by a facet, so as to cover the angle range defined in the regulations previously described. The second part is first reflected by a parabolic mirror towards another area of the side reflector and then scattered like the first part. This construction enables to gather most of light emitted from the LED and to redirect it to the angle domain defined within regulations.

Monte-carlo ray-tracing simulations were carried out for this reflector system, including accurate ray model for the LED source (a luxeon Rebel from Philips Lumileds). Figure 5 shows a result in terms of luminous intensity distribution.

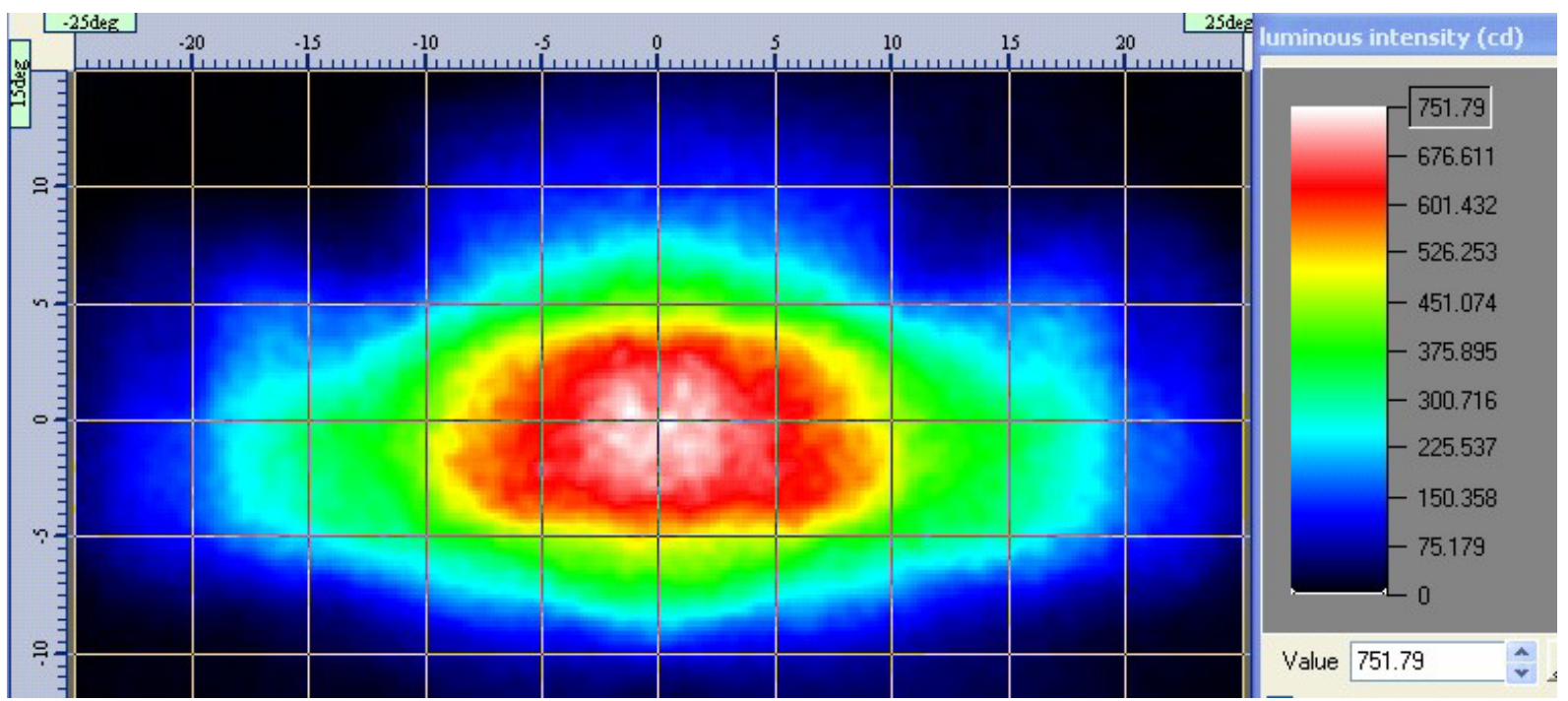

Figure 5: Simulated luminous intensity distribution 
For the sake of completion, let us precise that this part is associated with a housing and a transparent plastic cover, and that the whole part is then inserted into the car front bumper. Figure 6 shows from left to right a model of the whole part, a picture of the part integrated in a bumper, and the day-time running lamp as part of the SLK version 2011.
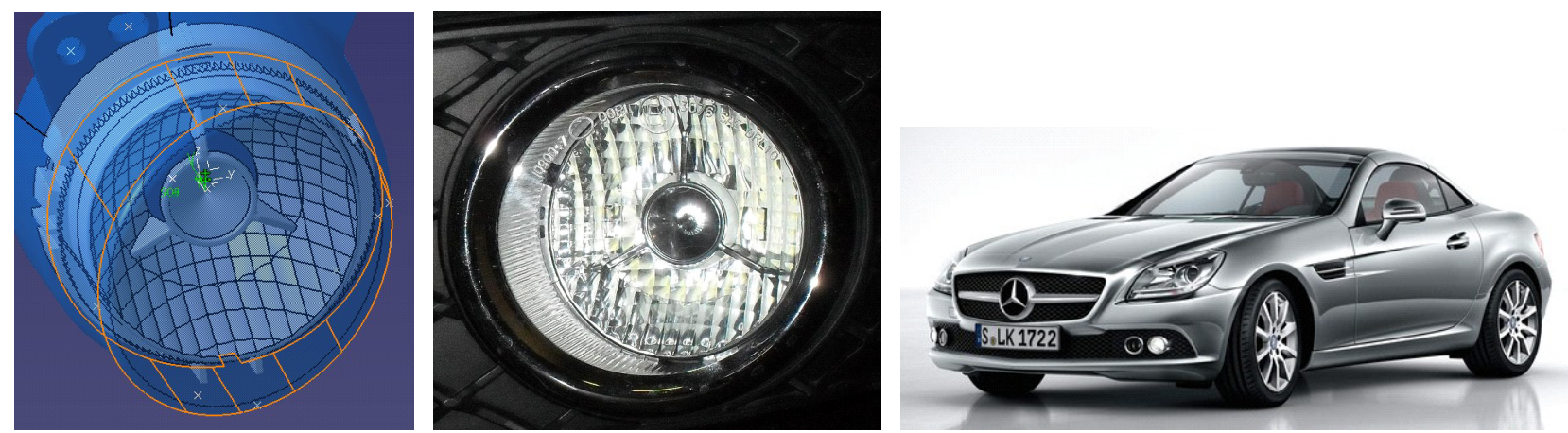

Figure 6: Part model (left), part in car bumper (center), and in an SLK Version 2011 (right)

\section{ELECTRONIC DESIGN}

Electronic design constitutes the second aspect of our energy saving efforts. Optical efficiency could be defined in terms of light collected with respect to light emitted from the LED source. This definition leads to nearly $100 \%$-efficiency for the proposed concept. In order to keep system efficiency as high as possible, it is necessary to ensure lowest possible energy lost when driving the light source.

There are several ways of driving LEDs ${ }^{6}$, each of them showing different efficiencies for different LED configurations (number, arrangement ...). Solutions may consist in direct resistor driving, current source driving, or DC-DC converter. The selected solution consists in a buck converter, and offers an efficiency of approximately $80 \%$, defined as the ratio of the electrical power used by the LED over the total power used by the part.

As for every electronic part, thermal aspects have to be considered in advance. Two main issues should retain our attention specially dealing with LEDs. First, at room temperature (around $23^{\circ} \mathrm{C}$ ), thermal degradation of LED luminous flux should remain as low as possible to comply with legal requirements. Measurements on prototype printed circuit boards (PCB) show a luminous flux reduction of the order of $10 \%$, what constitutes an acceptable result. Secondly, it has to be ensured that the conceived electronic design will survive to exterior environment conditions. For an automotive part placed in the car bumper, temperatures as high as $85^{\circ} \mathrm{C}$ can be reached, for instance in the situation of a car standing in traffic jam. Corresponding simulations and measurements were carried out to verify that the different electronic components, and particularly the LED PN-junction, would not be damaged under those circumstances.

Figure 7 shows the simulated temperature distribution within the part for an ambient temperature of $85^{\circ} \mathrm{C}$. 


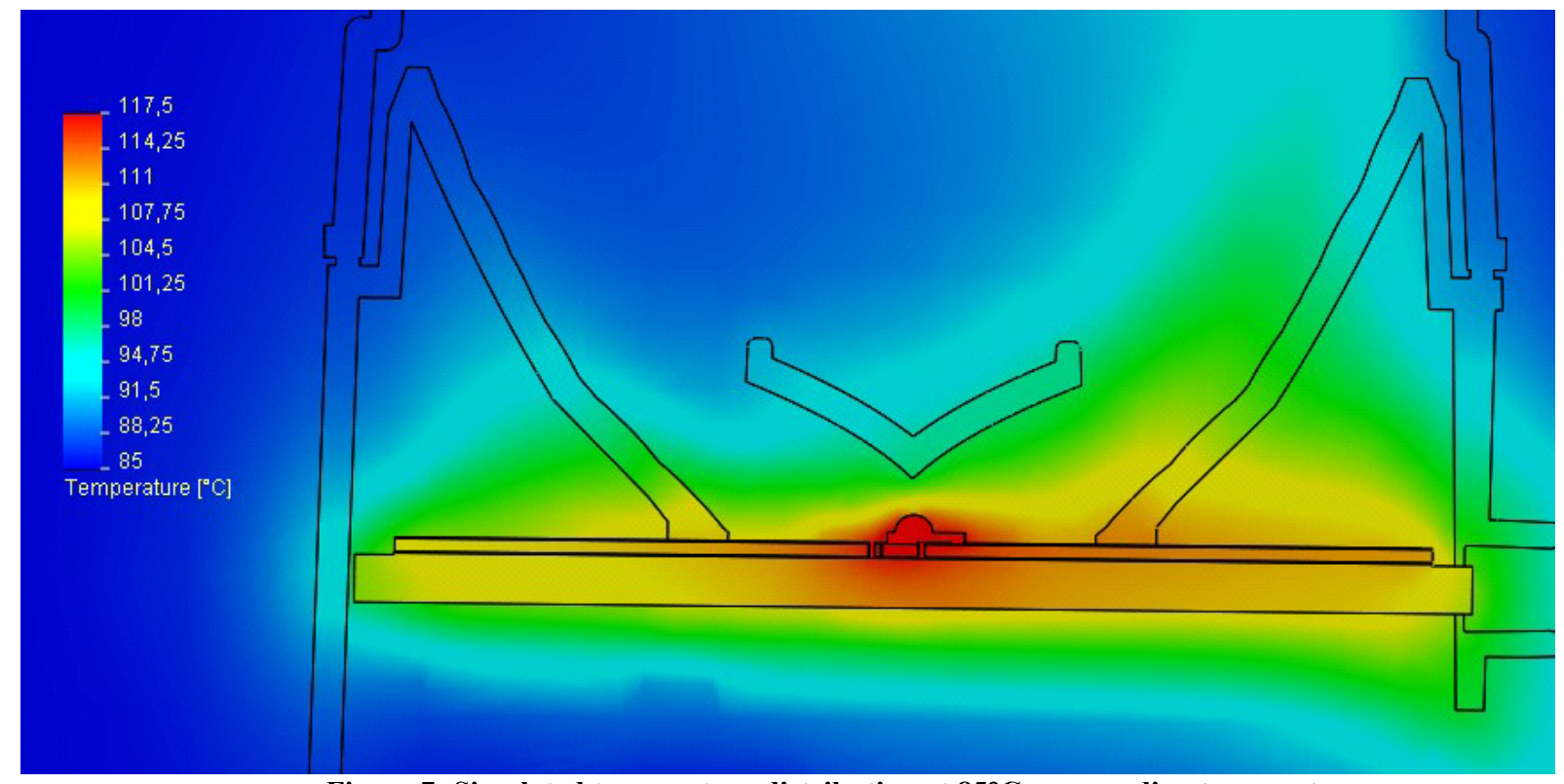

Figure 7: Simulated temperature distribution at $85^{\circ} \mathrm{C}$ surrounding temperature

\section{REAR FOG LAMP WITH LED}

Other signaling functions can also benefit from the use of LEDs, although their statistical use lies clearly below that of day-time running lamp. This last section intends to illustrate this possibility.

Rear fog lamp is statistically one of the functions with the shortest working time. The initial concept for SLK vehicle made use of LEDs for stop/tail and turn indicator functions, and of an incandescent bulb for reverse and rear fog lamps (lower right corner on Figure 8). The fog lamp would have had a power consumption of $24 \mathrm{~W}$ per vehicle and would have made necessary using heat resistant plastic materials. Another drawback would have been the resulting reduced stop/tail function, which could not be displayed till the inboard limit of the rear lamp. Figure 8 shows the initial design for SLK vehicles' rear lamp.

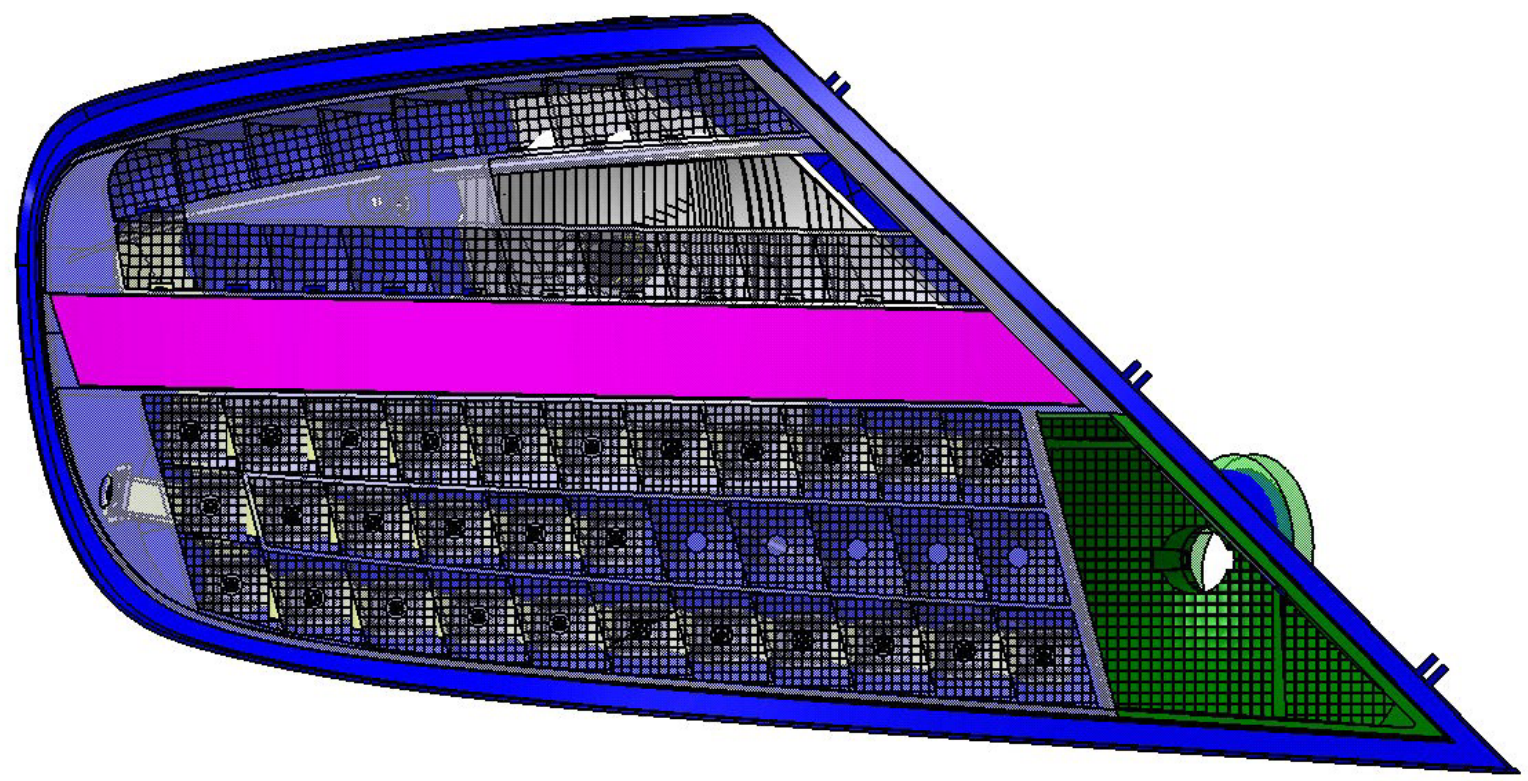

Figure 8: Initial design of SLK rear lamp including bulb-based rear fog lamp (right) 
We then developed a LED-based system to compete with the initial concept. It makes use of 4 LEDs (Osram LAG6SP) distributed on both sides of a common PCB. The PCB is then mounted horizontally, so that two LEDs emit light upward, and two downward respectively. The optical reflectors that are then positioned on top of it and below it can optimally gather light in a so-called "indirect" configuration (LEDs are not directly visible in front view). The final system presents an enhanced luminance due to its reduced illuminated surface, enables the stop/tail function to be prolonged till the inboard limit of the rear lamp, and last but not least a power consumption reduced down to $2 \mathrm{~W}$ per vehicle.

Figure 9 shows the final design for SLK vehicles' rear lamp.

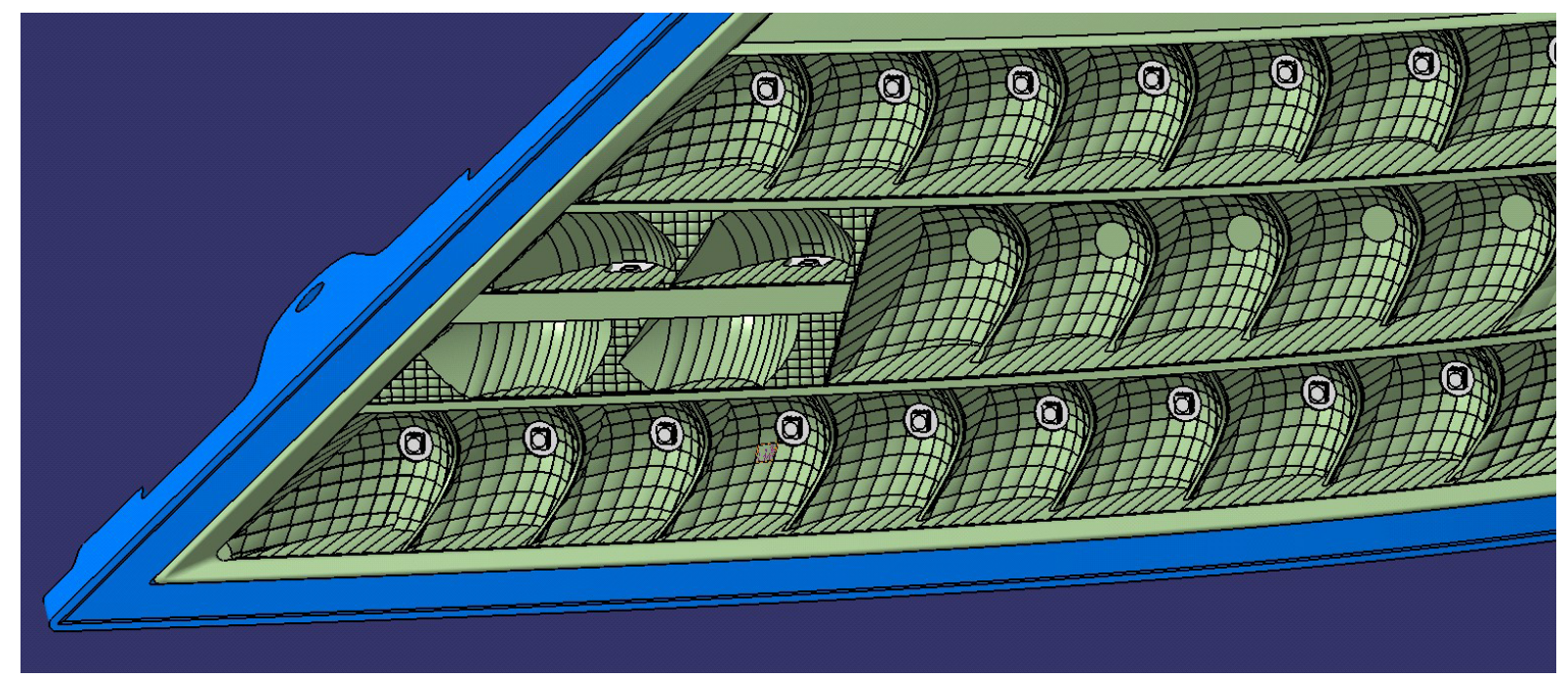

Figure 9: Final SLK rear lamp including LED-based rear fog lamp

As for the previously presented day-time running lamp, this rear fog lamp concept can be potentially implemented in other vehicles while keeping the working principles similar (i.e. same optical and electronic concepts).

\section{CONCLUSION}

Automotive exterior lighting remains a safety actor on traffic roads, ensuring both best vision possibilities during night drive and necessary vehicle visibility towards other traffic participants. LED-technology generally enables saving energy while offering equivalent or higher functionality. However, present optical systems are not necessarily targeted on efficiency, but rather a compromise with design. Use of energy optimized optical concepts together with adequate electronic LED driving enables further reduction of power consumption. This has led to the first day-time running lamp with a total power consumption below $5 \mathrm{~W}$ per vehicle. Other functions can benefit from clever use of LEDs, even signaling functions with low statistical working time.

\section{REFERENCES}

[1] Hamm, M., "Down with CO2-emissions - Ecologic potential and limits with lighting functions", Proc. ISAL, 1-10 (2009).

[2] Schoettle, B., Sivak, M., Fujiyama, Y., "LEDs and power consumption of exterior automotive lighting: implications for gasoline and electric vehicles", Proc. ISAL, 11-20 (2009).

[3] United Nations Economic Commission for Europe, "Vehicle regulation 37", www.unece.org.

[4] Schubert, E. F., [Light Emitting Diodes], Cambridge University Press (2006).

[5] United Nations Economic Commission for Europe, "Vehicle regulation 87", www.unece.org.

[6] Van der Broeck, H., Sauerländer, G., Wendt, M., "Power driver topologies and control schemes for LEDs", IEEE

Proc. APEC, 1319-1325 (2007). 